\title{
Size effect of the surface energy density of nanoparticles
}

\author{
Yin Yao, Yaochi Wei, Shaohua Chen * \\ LNM, Institute of Mechanics, Chinese Academy of Sciences, Beijing 100190, China
}

\section{A R T I C L E I N F O}

\section{Article history:}

Received 24 October 2014

Accepted 23 January 2015

Available online 29 January 2015

\section{Keywords:}

Metallic nanoparticles

Size effect

Lattice contraction

Surface energy density

\begin{abstract}
A B S T R A C T
The surface energy density of nanoparticles exhibits an obviously size-dependent behavior. However, how the surface energy density changes with the diameter of nanoparticles is still ambiguous. Based on a recently developed continuum theory considering the size effect in nanomaterials, theoretical analysis is carried out for various fcc metallic nanoparticles. Surface lattice contractions of nanoparticles are predicted and compared with the existing experimental data. As a result, the surface energy density decreases with the increase of nanoparticle diameter. Such a variation trend of surface energy density is contrary to the prediction of existing theoretical models but well consistent with the previously atomistic simulations and density functional calculations. The results in this paper provide a further understanding of the surface effect of nanoparticles, which should be helpful for the design of nanoscale devices or nanomaterials related to nanoparticles, such as NEMs and nanoparticlereinforced composites.
\end{abstract}

(c) 2015 Elsevier B.V. All rights reserved.

\section{Introduction}

As one of the typically nano-sized elements, nanoparticles find their potential applications as building blocks in many miniature devices such as conductors for flexible electronics [1], drug and gene delivery vehicles [2] and reinforcing phases in advanced nanocomposites [3]. The physical and chemical properties of nanoparticles, e.g., the surface energy density [4], melting temperature [5], chemical reactivity [6], and so on, are obviously different from the bulk counterparts and exhibit a distinctly size-dependent behavior. Such a special characteristic mainly arises from the large surface-tovolume ratio [7].

Surface free energy density is the most importantly physical attribute characterizing the nature of surface effect, which can be interpreted as a reversible work per unit area involved in creating a new surface [8]. For nanoparticles, a strong size effect of surface energy density emerges when the particle diameter is less than a few nanometers $[9,10]$. Due to the small size of nanoparticles, it is very difficult to measure the surface energy density experimentally. Theoretical models and numerical calculations are always being used to study the surface energy density at nanoscale. However, two kinds of different opinions exist.

From the thermodynamic perspective, a series of theoretical models have been developed to characterize the size effect of surface energy density [10-12], in which the surface energy density of fcc metallic

\footnotetext{
* Corresponding author. Tel.: +86 10 82543960; fax: + 861082543977

E-mail address: chenshaohua72@hotmail.com (S. Chen).
}

nanoparticles has a similar form to the classical Tolman's equation charactering the size effect of surface tension in nano-droplets [13],

$\frac{\phi}{\phi_{0 b}}=\frac{1}{1+\frac{4 \delta}{d}} \approx 1-\frac{4 \delta}{d}$

where $\phi$ and $\phi_{0 b}$ represent the surface energy densities of nanoparticles and bulk materials, respectively. $d$ is the nanoparticle diameter. $\delta$ is the Tolman length with the same order of magnitude as an atomic diameter of nanoparticles [10]. As a result, the surface energy density $\phi$ is predicted to increase monotonically with the increase of nanoparticle diameter and gradually tends to the bulk one $\phi_{0 b}$, which makes fcc metallic nanoparticles similar to liquid droplets [14]. The same conclusion has been made by Ouyang et al. [15,16] and a recent MD simulation with a modified analytic embedded atom method (MAEAM) for nickel nanoparticles [17].

Another group of investigations proposed a contrary opinion about the size effect of nanoparticle surface energy density. With the help of the Young-Laplace equation [18], surface energy densities of Ag and Pd nanoparticles were deduced based on the experimental data of lattice parameters [19-21], where the surface energy density of nanoparticles was found much larger than those of the bulk materials. Nanda et al. [22,23] studied further the surface energy density of Ag nanoparticles using experimental data related to the evaporation temperature, in which a surface energy density $7.2 \mathrm{~J} / \mathrm{m}^{2}$ was reported, close to $6.4 \mathrm{~J} / \mathrm{m}^{2}$ deduced from lattice contraction experiments [19], but much larger than the bulk one $1.2-1.4 \mathrm{~J} / \mathrm{m}^{2}$ [24]. A theoretical prediction [25] by analyzing the cohesive energies among atoms in $\mathrm{Ag}$ and Au nanoparticles gave consistent results with Nanda et al. [23]. However, the detailed 
size-dependent feature of surface energy density was not considered in the above literatures. Recently, a series of numerical calculations based on the density functional theory (DFT) and EAM potential found that surface energy densities of $\mathrm{Ag}$, $\mathrm{Al}$ and $\mathrm{Cu}$ nanoparticles decrease monotonically with the increase of nanoparticle diameters and finally approach the bulk values [26-28].

Which one of the above two viewpoints is true is still not clear. A feasible approach to study the size effect of nanoparticle surface energy density is to combine experimental data of size-dependent lattice contractions [19,21,29-31] with the continuum mechanics model. Such a technique has been adopted by many existing researches. The YoungLaplace equation was used to describe spontaneous changes of nanoparticle lattice parameters, however, in which the surface energy density was taken as a constant without considering effects of the particle size and surface deformation [9,32,33]. Wolfer [34] applied the surface elasticity theory [35] to fcc metallic nanoparticles, and the surface elastic constants were determined directly by fitting the experimental data with theoretical curves. The surface energy density of nanoparticles was formulated in terms of lattice strains, but the size effect was not considered also [34].

Recently, an elastic theory for nanomaterials and nanostructures has been developed by Chen and Yao [36], in which the dependence of the surface energy density on nanoscale characteristic sizes and surface residual deformations (surface relaxation) is physically described without involving the surface elastic constants. The new theory is used in the present paper in order to analyze the size effect of the surface energy density of several fcc metallic nanoparticles. Qualitative and quantitative comparisons among the theoretical predictions, existing calculations and experimental data are carried out. The size-dependent characteristic of the surface energy density of nanoparticles is analyzed and discussed. All the analyses provide a better understanding of the surface effect in nanoparticles and should be helpful for the design of novel nano-devices and nanomaterials.

\section{Brief introduction of the new theory for nanomaterials}

An elastic theory based on the surface energy density to characterize the size effect in nanomaterials has been proposed by Chen and Yao [36]. Consider a nano-solid with an initial (or reference) configuration in a three-dimensional Euclidean space, which transforms into a current configuration under an external loading. Assuming that the material has an idealized crystal structure, a Lagrangian coordinate system is imbedded in the deformed surface and attached to the atoms [37], with the principal axes 1 and 2 parallel to the two basic vectors of the surface unit cell, as shown in Fig. 1. $a_{01}$ and $a_{02}$ represent the lattice lengths in the two principal directions, respectively. $\beta$ denotes an angle between the two basic vectors. Due to a spontaneous surface relaxation, the lattice lengths become $a_{r 1}$ and $a_{r 2}$. Under an external loading, the lattice lengths further become $a_{1}$ and $a_{2}$ in the current configuration. The Lagrangian surface energy density $\phi_{0}$ in the reference configuration can be divided into a structural part $\phi_{0}^{\text {stru }}$ related to the surface strain energy and a chemical part $\phi_{0}^{\text {chem }}$ originating from the surface dangling-bond energy,

$$
\begin{aligned}
\phi_{0}= & \phi_{0}^{\text {stru }}+\phi_{0}^{\text {chem }} \\
\phi_{0}^{\text {stru }}= & \frac{E_{b}}{2 \sin \beta} \sum_{i=1}^{2} a_{0 i} \eta_{i}\left\{\left[3+\left(\xi_{i}+\xi_{i} \varepsilon_{s i}\right)^{-m}-3\left(\xi_{i}+\xi_{i} \varepsilon_{s i}\right)\right]\right. \\
& \left.\times\left[\xi_{i}^{2} \varepsilon_{s i}^{2}+\left(\xi_{i}-1\right)^{2}+2 \xi_{i}\left(\xi_{i}-1\right) \varepsilon_{s i}\right]\right\} \\
\phi_{0}^{\text {chem }}= & \phi_{0 b}\left(1-w_{1} \frac{D_{0}}{D}\right), \quad \eta_{1}=a_{01} / a_{02}, \quad \eta_{2}=a_{02} / a_{01}
\end{aligned}
$$

where $\phi_{0 b}$ is the bulk surface energy density and $D_{0}$ is a critical size $\left(D_{0}=3 d_{a}\right.$ for nanoparticles and $2 d_{a}$ for nano-thin films, where $d_{a}$ is the atomic diameter). $D$ is a characteristic scale of nanomaterials (e.g., thickness, diameter, etc.). $w_{1}$ is a parameter governing the size-dependent behavior of $\phi_{0}^{\text {chem }}$, which will be discussed in the following text. $E_{b}$ is the bulk Young's modulus, $\xi_{i}=a_{r i} / a_{0 i}$ denotes the surface relaxation parameter, $\varepsilon_{s i}=\left(a_{i}-a_{r i}\right) / a_{r i}$ is the surface strain induced only by the external loading and $m$ is a parameter describing the dependence of bond lengths on the binding energy ( $m=-4$ for alloys or compounds and $m=1$ for pure metals) [38].

The relationship between the Eulerian surface energy density $\phi$ in the current configuration and the Lagrangian surface energy density $\phi_{0}$ in the reference configuration satisfies the following equation:

$\phi=\frac{\phi_{0}}{J_{s}}$

where $J_{s}$ is a Jacobean determinant characterizing the surface deformation from the reference configuration to the current one. Eq. (3) can also be found in Nix and Gao [37] and Huang and Wang [39].

In the current configuration, the potential energy function $\Pi$ of nano-solids can be written as

$$
\Pi(\mathbf{u})=\int_{V-S} \psi(\boldsymbol{\varepsilon}) d V+\int_{S} \phi d S-\int_{V-S} \mathbf{f} \cdot \mathbf{u} d V-\int_{S_{p}} \mathbf{p} \cdot \mathbf{u} d S
$$

where $\psi$ is the elastic strain energy density, $\mathbf{f}$ and $\mathbf{p}$ are the body force and external surface traction; $\mathbf{u}$ and $\boldsymbol{\varepsilon}$ are the displacement and strain induced by $\mathbf{f}$ and $\mathbf{p}$. Variational analysis of Eq. (4) yields the equilibrium equation and stress boundary conditions,

$$
\left\{\begin{array}{l}
\boldsymbol{\sigma} \cdot \nabla+\mathbf{f}=0 \quad(\text { in } V-S) \\
\mathbf{n} \cdot \boldsymbol{\sigma} \cdot \mathbf{n}=\mathbf{p} \cdot \mathbf{n}-\gamma_{n} \mathbf{n} \quad(\text { on } S) \\
(\mathbf{I}-\mathbf{n} \otimes \mathbf{n}) \cdot \boldsymbol{\sigma} \cdot \mathbf{n}=(\mathbf{I}-\mathbf{n} \otimes \mathbf{n}) \cdot \mathbf{p}-\boldsymbol{\gamma}_{t} \quad(\text { on } S)
\end{array}\right.
$$

where $\boldsymbol{\sigma}$ is the bulk Cauchy stress tensor, $\mathbf{n}$ is the unit normal vector perpendicular to the boundary surface $S$ of the nano-solid, I is a unit tensor and $\gamma_{n}$ and $\boldsymbol{\gamma}_{t}$ are the normal and tangential components of an additional surface-induced traction vector, respectively, which characterizes

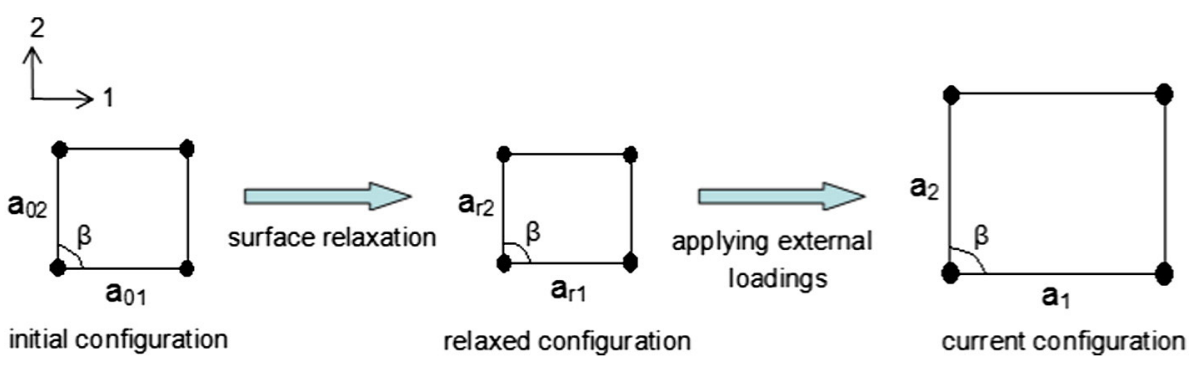

Fig. 1. Schematic of a surface unit cell in the initial (reference), relaxed and current configurations. 
the force disturbance at boundaries due to the surface effect. Based on an infinitesimal element, the virtual work method yields the surfaceinduced traction [36],

$\boldsymbol{\gamma}_{t}=\nabla_{s} \phi, \quad \gamma_{n} \mathbf{n}=\phi\left(\frac{1}{R_{1}}+\frac{1}{R_{2}}\right) \mathbf{n}=\phi\left(\mathbf{n} \cdot \nabla_{s}\right) \mathbf{n}$

where $\nabla_{s}$ is a surface gradient operator, $R_{1}$ and $R_{2}$ are the principal radii of curvature of a curved surface. Combining Eqs. (3), (5) and (6) leads to the governing equations of the new theory [36],

$$
\left\{\begin{array}{l}
\boldsymbol{\sigma} \cdot \nabla+\mathbf{f}=0 \quad(\text { in } V-S) \\
\mathbf{n} \cdot \boldsymbol{\sigma} \cdot \mathbf{n}=\mathbf{p} \cdot \mathbf{n}-\frac{\phi_{0}\left(\mathbf{n} \cdot \nabla_{s}\right)}{J_{S}} \quad(\text { on } S) \\
(\mathbf{I}-\mathbf{n} \otimes \mathbf{n}) \cdot \boldsymbol{\sigma} \cdot \mathbf{n}=(\mathbf{I}-\mathbf{n} \otimes \mathbf{n}) \cdot \mathbf{p}+\frac{\phi_{0}\left(\nabla_{S} J_{S}\right)}{J_{S}^{2}}-\frac{\nabla_{s} \phi_{0}}{J_{S}}
\end{array}\right.
$$

In contrast to the G-M theory [35], the new theory no longer requires the surface elastic constants. The Lagrangian surface energy density $\phi_{0}$ in the reference configuration serves as a unique quantity characterizing the surface effect of nanomaterials, which depends on the bulk surface energy density and the relaxation parameter. Both parameters have clearly physical meanings and are very easy to determine through experiment and simple MD simulation.

\section{The surface effect of nanoparticles}

\subsection{Radial shrinkage}

Consider a spherical nanoparticle with its center at the origin of a rectangular coordinate $\left\{x_{1}, x_{2}, x_{3}\right\}$ as shown in Fig. 2. A spherical coordinate system $\{r, \theta, \varphi\}$ is also attached on the particle with the $\theta$ - and $\varphi$ directions tangential to the particle surface and the $r$-direction normal to the particle surface. The un-deformed nanoparticle with an initial radius $r_{0}$ is regarded as the reference configuration. After spontaneous relaxation, the radius of the nanoparticle shrinks to $r_{1}$, leading to a negative displacement $\Delta r=r_{1}-r_{0}$ in the radial direction.

The equilibrium equation of nanoparticles in the spherical coordinate system is

$\frac{\mathrm{d} \sigma_{r}}{\mathrm{~d} r}+\frac{2\left(\sigma_{r}-\sigma_{\theta}\right)}{r}=0$ where $\sigma_{r}$ and $\sigma_{\theta}$ are the radial and circumferential stress components, respectively, satisfying the following constitutive relations,

$\sigma_{r}=(\lambda+2 \mu) \varepsilon_{r}+\lambda\left(\varepsilon_{\theta}+\varepsilon_{\varphi}\right), \quad \sigma_{\theta}=\sigma_{\varphi}=(\lambda+2 \mu) \varepsilon_{\theta}+\lambda\left(\varepsilon_{r}+\varepsilon_{\varphi}\right)$

Here, $\varepsilon_{r}=\mathrm{d} u_{r} / \mathrm{d} r$ and $\varepsilon_{\theta}=\varepsilon_{\phi}=u_{r} / r$ represent the radial and circumferential strain components, respectively; $u_{r}$ is the radial displacement; and $\lambda$ and $\mu$ are the Lame constants of bulk materials.

After spontaneous relaxation, the traction boundary condition can be obtained as

$\left(\sigma_{r}\right)_{r=r_{1}}=-\gamma_{n}=-\phi(\mathbf{n} \cdot \nabla)=-\frac{2 \phi}{r_{1}}=-\frac{2 \phi}{r_{0}+\Delta r}$

Substituting Eq. (9) into Eqs. (8) and (10) yields

$\frac{\mathrm{d}^{2} u_{r}}{\mathrm{~d} r^{2}}+\frac{2}{r} \frac{\mathrm{d} u_{r}}{\mathrm{~d} r}-\frac{2 u_{r}}{r^{2}}=0, \quad\left[(\lambda+2 \mu) \frac{\mathrm{d} u_{r}}{\mathrm{~d} r}+\frac{2 \lambda u_{r}}{r}\right]_{r=r_{1}}=-\frac{2 \phi}{r_{1}}$

The solution of Eq. (11) is

$u_{r}=-\frac{2 \phi}{3 \kappa} \frac{r}{r_{1}}$

where $\kappa=\lambda+\frac{2 \mu}{3}$ is the bulk modulus of nanoparticles.

Then we have the circumferential strain components and radial shrinkage on the surface,

$\varepsilon_{\theta}^{s}=\varepsilon_{\varphi}^{s}=\left(\frac{u_{r}}{r}\right)_{r=r_{1}}=-\frac{2 \phi}{3 \kappa r_{1}}, \quad \Delta r=\left(u_{r}\right)_{r=r_{1}}=-\frac{2 \phi}{3 \kappa}$

where $\varepsilon_{\theta}^{s}, \varepsilon_{\varphi}^{s}$ are Eulerian surface strains in the current configuration. One can see that the radial displacement $\Delta r$ of the nanoparticle surface has a similar form to that obtained from the classical Young-Laplace equation [19,33].

Substituting Eq. (3) into (13) leads to

$\varepsilon_{\theta}^{s}=\varepsilon_{\varphi}^{s}=-\frac{2 \phi_{0}}{3 J_{s} \kappa r_{1}}, \quad \Delta r=-\frac{2 \phi_{0}}{3 J_{s} \kappa}$

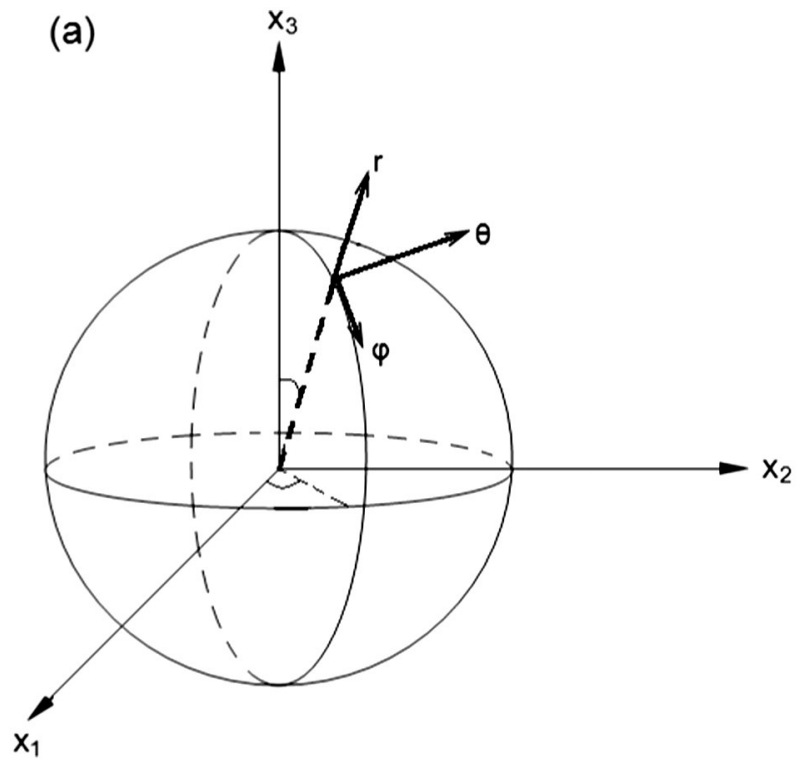

(b)

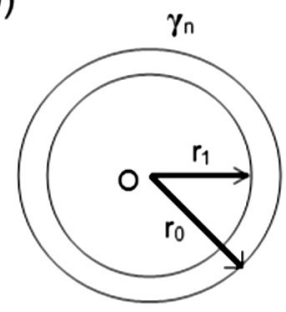

Fig. 2. Schematic of a perfectly spherical nanoparticle. (a) 3-D configuration; (b) radial shrinkage due to the lattice contraction. 
Table 1

Material parameters of various fcc metallic nanoparticles [15,19,21,29,30,40].

\begin{tabular}{lllcll}
\hline & $\phi_{\mathrm{bb}}(\mathrm{N} / \mathrm{m})$ & $a_{0}(\mathrm{~nm})$ & $E_{\mathrm{b}}(\mathrm{GPa})$ & $\kappa(\mathrm{GPa})$ & $d_{\mathrm{a}}(\mathrm{nm})$ \\
\hline $\mathrm{Ag}$ & 1.14 & 0.4086 & 78 & 100 & 0.2889 \\
$\mathrm{Au}$ & 1.5 & 0.4074 & 79 & 165 & 0.2884 \\
$\mathrm{Cu}$ & 1.79 & 0.3615 & 110 & 114 & 0.25 \\
$\mathrm{Pd}$ & 2.0 & 0.389 & 121 & 180 & 0.35 \\
$\mathrm{Pt}$ & 2.5 & 0.3916 & 168 & 230 & 0.36 \\
\hline
\end{tabular}

According to Eq. (2), it is interesting to find that the normal surface displacement in the new theory depends not only on the surface deformation but also on the particle size.

\subsection{The surface energy density of nanoparticles}

For a spherical nanoparticle experiencing only a spontaneous lattice contraction, the surface deformation is isotropic [34]. Imaging an undeformed nanoparticle bounded by $N$ square surface unit cells with atomic spacings $a_{01}=a_{02}=a_{0}$, where $a_{0}$ is the bulk lattice constant. After the isotropic surface relaxation, atomic spacings in the surface unit cells are changed from $a_{0}$ to $\xi a_{0}$ and the surface relaxation parameters in Eq. (2) become $\xi_{1}=\xi_{2}=\xi$, leading to the surface residual strain $\varepsilon_{\text {res }}=\xi-1$ and the Jacobean determinant $J_{s}=\left(1+\varepsilon_{\text {res }}\right)^{2}=\xi^{2}$.

The structural part of the Lagrangian surface energy density of an fcc metallic nanoparticle without loading can then be expressed as

$\phi_{0}^{\mathrm{stru}}=\frac{E_{b} a_{0}}{\sin \beta}\left(3+\frac{1}{\xi}-3 \xi\right)(\xi-1)^{2}$

The corresponding chemical part can be written as

$\phi_{0}^{\text {chem }}=\phi_{0 b}\left(1-c_{1} \frac{d_{a}}{d_{0}}\right) \approx \frac{\phi_{0 b}}{1+c_{1} \frac{d_{a}}{d_{0}}}$

where $d_{a}$ is the atomic diameter of the nanoparticle and $c_{1}=3 w_{1} ; d_{0}=$ $2 r_{0}$ is the diameter of the nanoparticle in the un-deformed configuration. It can be found that Eq. (16) is exactly the original form of Tolman's equation [13].

Thus, the Lagrangian surface energy density of an fcc metallic nanoparticle can be specially expressed as

$\phi_{0}=\frac{\phi_{0 b}}{1+c_{1} \frac{d_{a}}{2 r_{0}}}+\frac{E_{b} a_{0}}{\sin \beta}\left(3+\frac{1}{\xi}-3 \xi\right)(\xi-1)^{2}$

The size-dependent feature of $\phi_{0}$ is contributed from the chemical part [15], which is governed by the parameter $c_{1}$.

Compared to existing theoretical models [32-34], the surface energy density of nanoparticles in the present model depends not only on the particle size but also on the surface relaxation parameter.

\section{Analysis of the size effect of nanoparticle surface energy density}

For a nanoparticle bounded by $N$ surface unit cells, surface areas before and after lattice contraction satisfy

$\frac{S_{1}}{S_{0}}=\frac{N \xi^{2} a_{0}^{2} \sin \beta}{N a_{0}^{2} \sin \beta}=\xi^{2}=\frac{4 \pi r_{1}^{2}}{4 \pi r_{0}^{2}}=\frac{r_{1}^{2}}{r_{0}^{2}}$ which yields

$\xi-1=\frac{\Delta r}{r_{0}}=\frac{\Delta a}{a_{0}}$

Substituting Eq. (17) into Eq. (14) and noting the Jacobean determinant $J_{s}=\xi^{2}$ result in the governing equation characterizing the size effect for nanoparticles,

$\xi-1=-\frac{2}{3 \xi^{2} \kappa r_{0}}\left[\frac{\phi_{0 b}}{1+c_{1} \frac{d_{a}}{2 r_{0}}}+\frac{E_{b} a_{0}}{\sin \beta}\left(3+\frac{1}{\xi}-3 \xi\right)(\xi-1)^{2}\right]$

The surface relaxation parameter $\xi$ can be achieved from Eq. (20), which further leads to the lattice contraction $\Delta a / a_{0}$. Several kinds of fcc metallic nanoparticles are studied, such as $\mathrm{Ag}, \mathrm{Au}, \mathrm{Cu}, \mathrm{Pd}$ and Pt, with the material properties listed in Table 1 . The bulk surface energy density $\phi_{0 b}$ is taken from Sheng et al. [40]. The bulk lattice constant $a_{0}$ is from the existing experimental studies [19,21,29-31]. The Young's moduli, bulk moduli and atomic diameters have been given by Ouyang et al. [15] and Sheng et al. [40]. The value of parameter $c_{1}$ will take $4,2,1,-1,-2$ and -3 , respectively, in order to analyze why two opposite size-dependent behaviors were reported for the surface energy density of nanoparticles. Detailed discussions on the effect of parameter $c_{1}$ will also be made in the following text.

Lattice contractions of $\mathrm{Ag}, \mathrm{Au}, \mathrm{Cu}, \mathrm{Pd}$ and Pt nanoparticles obtained from electron diffraction micrographs $[19,21,29,30]$ are used to compare with our theoretical predictions as shown in Fig. 3a-e, respectively. One can see that no matter parameter $c_{1}$ is positive or negative, the lattice contraction of different metallic nanoparticles is always increasing with the decrease of nanoparticle diameters. For almost all the five kinds of different metallic nanoparticles, theoretical predictions with a negative $c_{1}$ are always better consistent with the experimental data than those with a positive $c_{1}$. An optimum range $-2 \sim-1$ for $c_{1}$ can be deduced from the quantitative comparisons. Deviations between the theoretical predictions and experimental data may be due to several factors, such as sample preparations, absorbed and dissolved impurities and measurement techniques [19,21,29,30].

According to Eq. (17), a negative $c_{1}$ will lead to a reducing surface energy density with the increase of nanoparticle diameters, and the surface energy density of nanoparticles must be larger than the bulk ones as shown in Fig. 4. Such a size-dependent behavior for nanoparticles is consistent well with the previously numerical results [26-28] but opposite to the existing theoretical predictions $[10,15,16]$. It suggests that the classical Tolman's equation for liquid nano-droplets $\left(c_{1}=4\right)$ may not be appropriate for solid nanoparticles [23]. Coincidentally, the chemical part of the present surface energy density with a negative $c_{1}$ is analogous to the Tolman's equation with a negative Tolman length [41-43].

The Lagrangian surface energy density of Ag nanoparticles predicted theoretically is further compared with the calculations using the density functional theory (DFT) [26] as shown in Fig. 4. One should be noted that the bulk surface energy density $\phi_{0 b}$ of $\mathrm{Ag}$ nanoparticles here is $1.0 \mathrm{~J} / \mathrm{m}^{2}$, which is taken from the DFT calculations [26]. It is found that, for a negative $c_{1}$, the surface energy density decreases with the increase of nanoparticle diameters, consistent well with the DFT calculations, while an increasing behavior is exhibited for a positive $c_{1}$, contrary to the variation trend of DFT simulations. Even in the cases with a negative $c_{1}$, a quantitative difference between theoretical results and DFT calculations is inevitable since the presently theoretical model assumes a perfectly spherical nanoparticle in contrast to a nanocluster with a polyhedral shape in DFT calculation model [26]. The shape difference should be responsible for the deviation between the theoretical and numerical results [12]. Both 

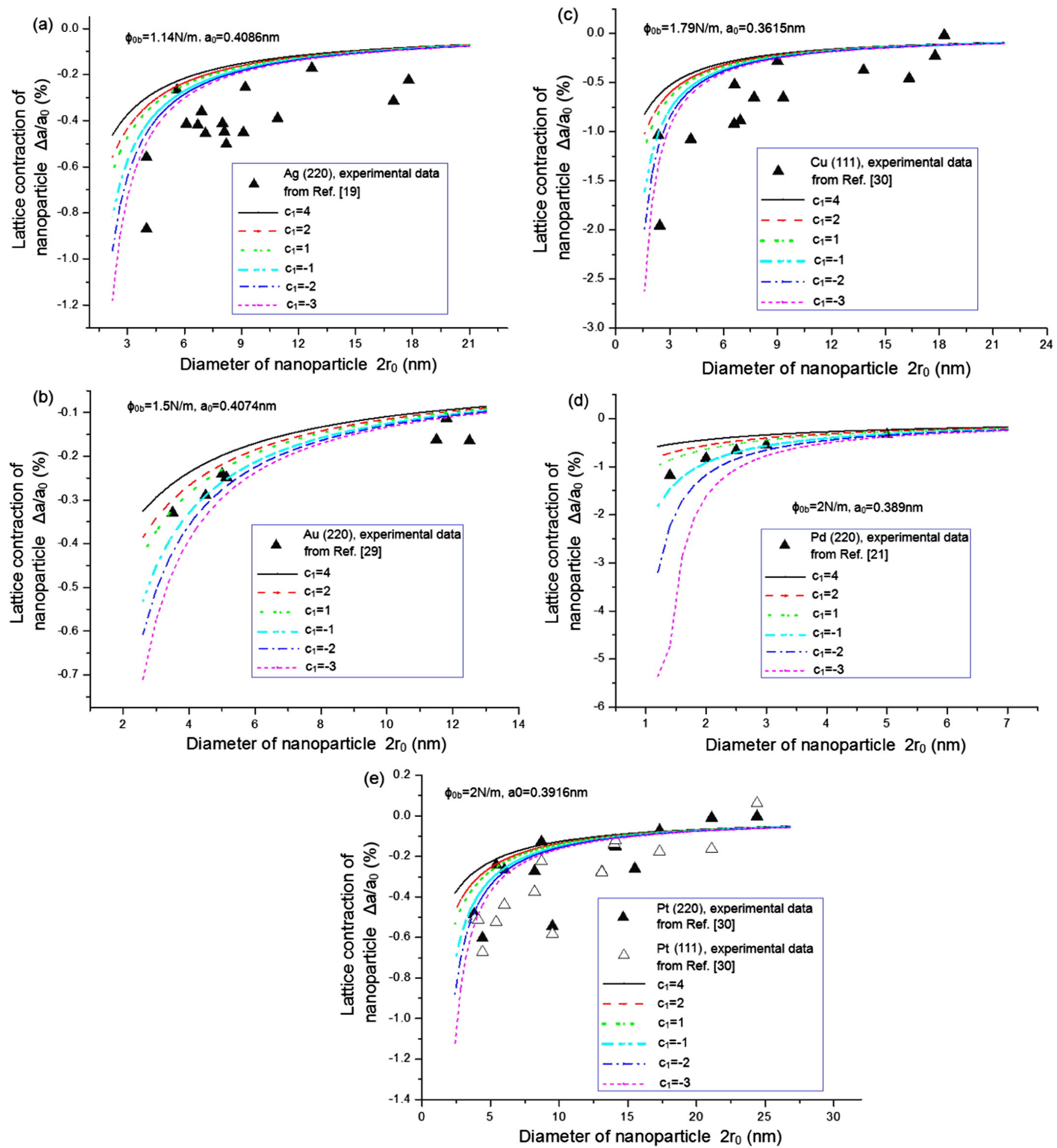

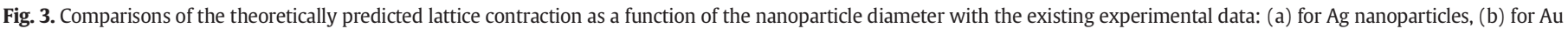
nanoparticles, (c) for Cu nanoparticles, (d) for Pd nanoparticles and (e) for Pt nanoparticles.

the theoretical predictions and the numerical computations of the normalized surface energy density tend to one when the diameter of nanoparticles is large enough.

\section{Conclusions}

Based on the recently developed continuum theory characterizing the surface effect in nanomaterials [36], the size effect of surface energy density of nanoparticles is investigated. The lattice contraction of different fcc metallic nanoparticles is first predicted theoretically and compared with the experimental data, leading to a negative coefficient in the chemical part of surface energy density. As a result, the surface energy density of nanoparticles is found to increase with the decrease of nanoparticle diameters, which is opposite to the existing theoretical predictions but consistent with the previously atomistic and DFT simulations. All the analyses in this paper should be helpful to clarify the ambiguous argument on the size-dependent behavior of nanoparticle surface energy density. 


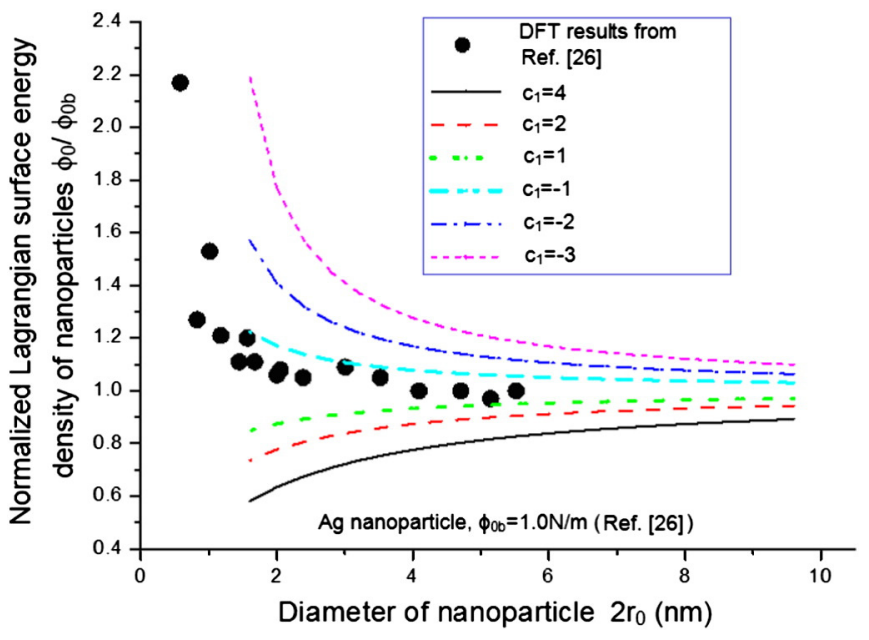

Fig. 4. Comparisons of the theoretically predicted surface energy density of Ag nanoparticles with DFT simulation results [26].

\section{Acknowledgments}

The work reported here is supported by the NSFC through grant nos. 11372317 and 11125211, the Nano-project (2012CB937500), and the CAS/SAFEA International Partnership Program for Creative Research Teams.

\section{References}

[1] D. Huang, F. Liao, S. Molesa, D. Redinger, V. Subramanian, J. Electrochem. Soc. 150 (7) (2003) 412 .

[2] A. Albanese, P.S. Tang, W.C.W. Chan, Annu. Rev. Biomed. Eng. 14 (2012) 1.

[3] G. Schmidt, M.M. Malwitz, Curr. Opin. Colloid Interface Sci. 8 (1) (2003) 103.

[4] K.K. Nanda, Phys. Lett. A 376 (19) (2012) 1647.

[5] H. Li, P.D. Han, X.B. Zhang, M. Li, Mater. Chem. Phys. 137 (3) (2013) 1007.
[6] C.N.R. Rao, G.U. Kulkarni, P.J. Thomas, P.P. Edwards, Chem. Eur. J. 8 (1) (2002) 28.

[7] F.D. Fischer, T. Waitz, D. Vollath, N.K. Simha, Prog. Mater. Sci. 53 (3) (2008) 481.

[8] J.W. Gibbs, Collected Works, Longmans, Green, and Company, New York, 1928. 314

[9] R. Shuttleworth, Proc. Phys. Soc. A 63 (5) (1949) 444.

[10] H.M. Lu, Q. Jiang, J. Phys. Chem. B 108 (18) (2004) 5617.

[11] Q. Jiang, H.M. Lu, Surf. Sci. Rep. 63 (10) (2008) 427.

[12] S.Y. Xiong, W.H. Qi, Y.J. Cheng, B.Y. Huang, M.P. Wang, Y.J. Li, Phys. Chem. Chem. Phys. 13 (2011) 10648.

[13] R.C. Tolman, J. Chem. Phys. 17 (3) (1949) 333.

[14] V.M. Samsonov, L.M. Shcherbakov, A.R. Novoselov, A.V. Lebedev, Colloids Surf. A Physicochem. Eng. Asp. 160 (2) (1999) 117.

[15] G. Ouyang, X. Tan, G.W. Yang, Phys. Rev. B 74 (19) (2006) 195408.

[16] G. Ouyang, C.X. Wang, G.W. Yang, Chem. Rev. 109 (9) (2009) 4221.

[17] W.H. Luo, W.Y.K.L. Hu, F.S. Liu Su, Appl. Surf. Sci. 265 (15) (2013) 375.

[18] T. Young, Philos. Trans. R. Soc. Lond. 95 (1805) 65.

[19] H.J. Wasserman, J.S. Vermaak, Surf. Sci. 22 (1) (1970) 164.

[20] P.A. Montano, W. Schulze, B. Tesche, G.K. Shenoy, T.I. Morrison, Phys. Rev. B 30 (2) (1984) 672.

[21] R. Lamber, S. Wetjen, N.I. Jaeger, Phys. Rev. B 51 (16) (1995) 10968.

[22] K.K. Nanda, A. Maisels, F.E. Kruis, H. Fissan, S. Stappert, Phys. Rev. Lett. 91 (10) (2003) 106102.

[23] K.K. Nanda, A. Maisels, F.E. Kruis, H. Fissan, S. Stappert, Phys. Rev. Lett. 92 (17) (2004) 179602.

[24] L. Vitos, A.V. Ruban, H.L. Skriver, J. Kollar, Surf. Sci. 411 (1-2) (1998) 186.

[25] D. Xie, M.P. Wang, L.F. Cao, Phys. Status Solidi 242 (8) (2005) 76.

[26] B. Medasani, Y.H. Park, I. Vasiliev, Phys. Rev. B 75 (2007) 235436

[27] B. Medasani, I. Vasiliev, Surf. Sci. 603 (13) (2009) 2042.

[28] J.J. Bian, G.F. Wang, X.Q. Feng, Acta Mech. Solida Sin. 25 (6) (2012) 557

[29] C.W. Mays, J.S. Vermaak, K.D. Wilsdorf, Surf. Sci. 12 (2) (1968) 134.

[30] H.J. Wasserman, J.S. Vermaak, Surf. Sci. 32 (1) (1972) 168.

[31] J. Woltersdorf, A.S. Nepijko, E. Pippel, Surf. Sci. 106 (1-3) (1981) 64.

[32] W.H. Qi, M.P. Wang, J. Nanoparticle Res. 7 (1) (2005) 51.

[33] Z.X. Huang, P. Thomson, S.L. Di, J. Phys. Chem. Solids 68 (4) (2007) 530.

[34] W.G. Wolfer, Acta Mater. 59 (20) (2011) 7736.

[35] M.E. Gurtin, A.I. Murdoch, Arch. Ration. Mech. Anal. 57 (4) (1975) 291.

[36] S.H. Chen, Y. Yao, ASME J. Appl. Mech. 81 (12) (2014) 121002.

[37] W.D. Nix, H.J. Gao, Scr. Mater. 39 (12) (1998) 1653.

[38] C.Q. Sun, Prog. Mater. Sci. 48 (6) (2003) 521.

[39] Z.P. Huang, J.X. Wang, Acta Mech. 182 (3-4) (2006) 195

[40] H.W. Sheng, M.J. Kramer, A. Cadien, T. Fujita, M.W. Chen, Phys. Rev. B 83 (13) (2011) 134118.

[41] M. Iwamatsu, J. Phys. Condens. Matter 6 (13) (1994) 173.

[42] A.E. Giessen, E.M. Blokhuis, J. Chem. Phys. 131 (16) (2009) 164705.

[43] G. Graziano, Chem. Phys. Lett. 497 (1-3) (2010) 33. 